\title{
Damage at a tungsten surface induced by impacts of self-atoms
}

\author{
Yong $\mathrm{Wu}^{1}$, Predrag Krstic ${ }^{2, *}, \mathrm{Fu}$ Yang Zhou ${ }^{3}$, and Fred Meyer ${ }^{4}$ \\ ${ }^{1}$ Data Center for High Energy Density Physics, Institute of Applied Physics and \\ Computational Mathematics, P. O. Box 8009, Beijing 100088, P. R. China \\ ${ }^{2}$ Institute for Advanced Computational Science, Stony Brook University, Stony Brook, NY \\ 11794-5250, USA \\ ${ }^{3}$ College of Material Sciences and Optoelectronic Technology, \\ University of the Chinese Academy of Sciences, PO Box 4588, Beijing 100049, China \\ ${ }^{4}$ Physics Division, Oak Ridge National Laboratory, Oak Ridge, TN 37831-6372 USA
}

\begin{abstract}
We study evolution of the surface defects of a $300 \mathrm{~K}$ tungsten surface due to the cumulative impact of $0.25-10 \mathrm{keV}$ self-atoms. The simulation is performed by molecular dynamics with bond-order Tersoff-form potentials. At all studied impact energies the computation shows strong defect-recombination effect of both created Frenkel pairs as well as recombination of the implanted atoms with the vacancies created by the sputtering. This leads to a saturation of the cumulative count of vacancies as long as the implantation per impact atom exceeds sputtering and to a saturation of the interstitial count when production of the sputtered particles per impact atom becomes larger than 1 (at sufficiently high energies). The number of cumulative defects is fitted as functions of impact fluence and energy, enabling their analytical extrapolation outside the studied range of parameters.
\end{abstract}

PACS: 34.35.+a , 52.55.Pi, 52.55.Rk, 79.20.Rf

*Corresponding author e-mail: predrag.krstic@stonybrook.edu

(C) 2015. This manuscript version is made available under the Elsevier user license http://www.elsevier.com/open-access/userlicense/1.0/ 


\section{Introduction}

The next-generation fusion reactors will be characterized by high fluxes of neutrons and plasmaparticles at all plasma-facing components, and could operate with hot walls for thermodynamic efficiency of the energy conversion. Tungsten is favorite for the wall material due to its excellent thermo-mechanical properties, primarily low erosion rate, high melting temperature and low hydrogen retention. The D-T fusion produces $3.5 \mathrm{MeV}$ alpha-particles and 14.1 MeV neutrons whose effects to the tungsten walls are still being evaluated. Of particular interest is growth of so-called tungsten "fuzz" [1, 2], appearing above critical fluences, fluxes, energies and temperatures. The effect of damage in tungsten caused by $14.1 \mathrm{MeV}$ neutrons on the interactions of $\mathrm{D}, \mathrm{T}$ and He with the material is still being intensively studied. Impact of self-atoms on the tungsten surface is often used as a "surrogate" for the damage inflicted by neutrons, since selfions or -atoms of appropriate impact energies can represent the Primary Knock-on Atoms (PKA) created by impacting neutrons in a burning fusion reactor that start the damage cascades in the first wall of magnetic fusion devices.

The surface of the plasma facing material is exposed primarily to $\mathrm{D}$, and $\mathrm{T}$, the dominant constituents of the fusion plasma. Although tungsten has much lower solubility for hydrogen [3] than carbon, the phenomena of super-saturation and deep permeation of $\mathrm{H}$ in tungsten can cause bubble, blister and crack formation in the material under high fusion reactor particle [3-5]. If not well understood and controlled, these features might raise questions regarding the choice of tungsten as a good fusion material [3].

It has been known that defects in tungsten, in particular at the grain boundaries and in vacancies, as well as presence of extraneous polluting particles (like O, C), are preferred sites for deuterium and helium retention $[3,6]$. While the grain structure is mainly a consequence of the 
material metallurgy, other material defects are created by pre-bombardment by various energetic species, which can stimulate the formation of He-bubbles in tungsten and even the growth of $\mathrm{He}$ "fuzz" $[1,2,7,8]$, although the effect of near-surface radiation damage on nanofuzz formation still remains to be elucidated. Most of the studies of neutron-induced damage use self-ion/atom irradiation, which can conveniently produce significant damage in the near-surface region $[9,10]$. In preparation for simulations of plasma-surface interactions (erosion, hydrogen and helium uptake and recycling) occurring in tungsten walls damaged by energetic neutrons, we are thus studying the evolution dynamics of vacancies and interstitials to relatively high dpa doses of tungsten surfaces bombarded by self-atoms.

Single impact MD simulations of displacement damage in a variety of bcc and fcc metals and in a wide range of temperatures and impact energies has been extensively investigated [1115]. Thus, Bacon [12] reviewed evolution of displacement cascade in a bulk, formed by PKA with kinetic energy larger than a few hundred $\mathrm{eV}$, not seeing significant effects of a particular crystal lattice. He considers two stages developing in time: The collision phase (a few tenths of ps) in which energy of PKA is distributed by multiple collisions to many atoms which leave their lattice sites. In the second phase (several ps) the majority of the displaced atoms of the disordered core (or liquid phase) return to their lattice sites by thermal relaxation. The atoms that are not able to regain their lattice sites become SIA (self-interstitial atoms) at the periphery of the core, creating together with vacant sites the primary damage state. As discussed by Nordlund [13], for the case of copper and nickel, the separation of interstitials and vacancies, present in the contraction phase of the liquid core formed by cascades inside the material, is particularly efficient at the surface, producing adatom islands far from the liquid zone of the cascade. Stoller [14] considered formation and size of both vacancy and interstitial clusters during a displacement 
intra-cascade in iron. The number of the surviving point defects and size of the clusters increases with increase of the cascade energy, following the NRT [12, 14, and references therein] power law, but weakly depend on the temperature. We extend such studies to simulations of the cumulative effects of displacement damage by following, in addition to the temporal evolution of the damage cascade launched by each self-atom impact, the accumulation of this damage during cumulative impacts of such atoms. Recent studies of Calder at al. [15] on iron substrate support the previous findings that the number of vacancies and SIAs that survive intra-cascade recombination is fairly insensitive to the metal and temperature.

We previously studied the evolution of vacancies and interstitials in a hot tungsten surface, as well its erosion, during successive impacts of self-atoms $[9,16]$. The present work is a continuation of that effort to study cumulative effects in plasma-material interactions near surface under the extreme conditions of high-radiation damage and high temperature projected for next-generation fusion reactors. Considering the evolution of a single cascade, our findings are in agreement with previous works, such as fast creation of the defect core, after which most of displaced atoms return to their sites in the ps-time range, as shown in Figs. 1a, and 1b.
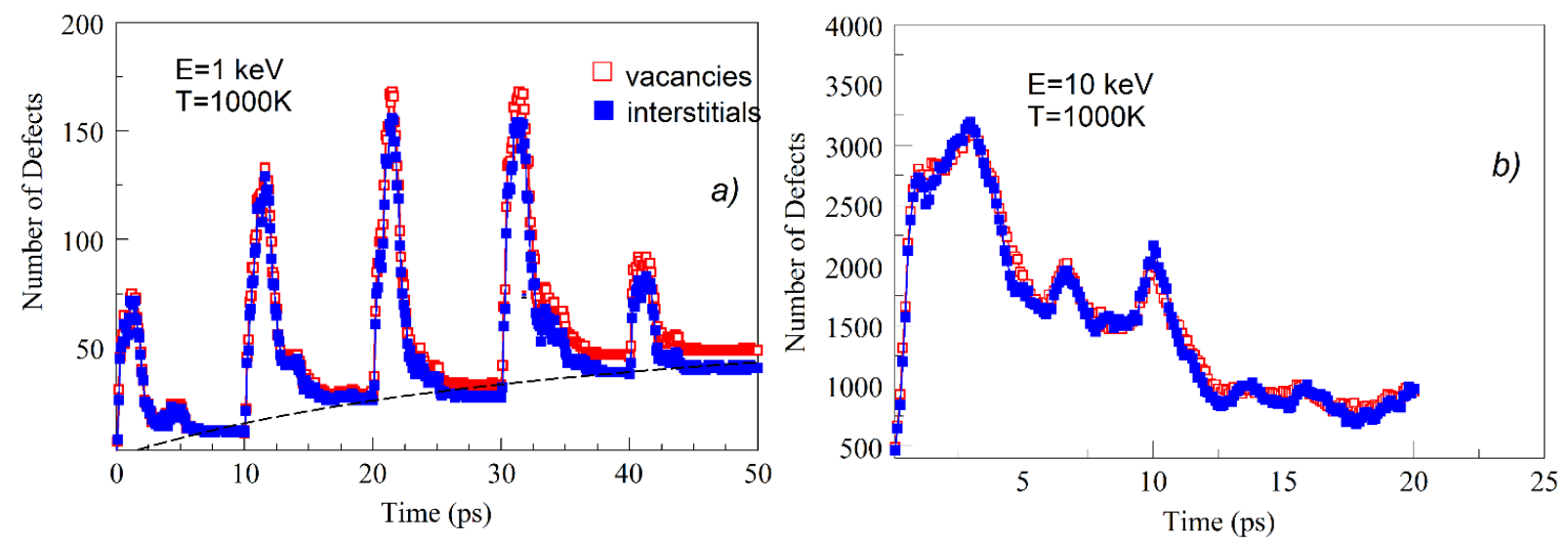

Figure 1.(a) Evolution of defects in a few 10ps impact cascades at $1 \mathrm{keV}$, The dashed line is following the survived defects.(b) Evolution of one cascade at $10 \mathrm{keV}$ impact, with longer recombination time. 
The clustering of vacancies and interstitials at the surface of tungsten is visible even after a large number of impacts, as seen in Fig. 2, and as also discussed for $\mathrm{Cu}$ and $\mathrm{Ni}$ in [13].
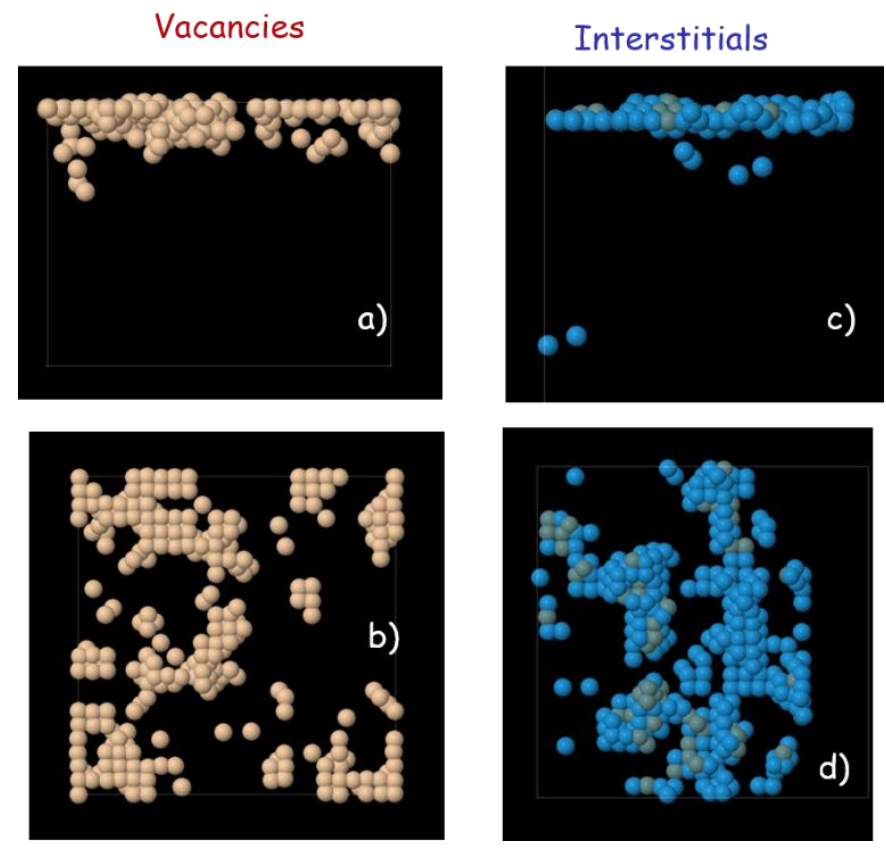

Figure 2. Clustering of vacancies and interstitials at the tungsten surface after 888 impacts of $1 \mathrm{keV}$ selfatoms on $1000 \mathrm{~K}$ tungsten. a) and c) are side views, clearly showing accumulation of vacancies and adatoms at the surface, while b) and d) are top views of the surface, showing the separated clustering of vacancies and interstitials. The majority of adatoms are from implanted self-atoms (blue), while SIA's are visible in ocker color.

The number of surviving defects is increasing with energy, and both dependence of the number of vacancies and interstitials follow the NRT power law for self-atom impact energy E in the range from $0.25 \mathrm{keV}$ to $6 \mathrm{keV}$, as shown in Fig. 3, obtained by averaging of the surviving defects in the first ten cascades. This power law dependence was -found also for Fe [14] and other metals [12] in a bulk, but in a different range of energies and with different exponents. Our findings are likely due to the effect of the surface, which significantly influences the survival probability. In addition, this dependence on PKA energy changes with accumulation of defects and will be considered in our forthcoming publication. While our previous theoretical study was limited to $1 \mathrm{keV}$ and $10 \mathrm{keV}$ self-atoms and hot $(1000 \mathrm{~K})$ tungsten surfaces $[9,16]$, in the present 


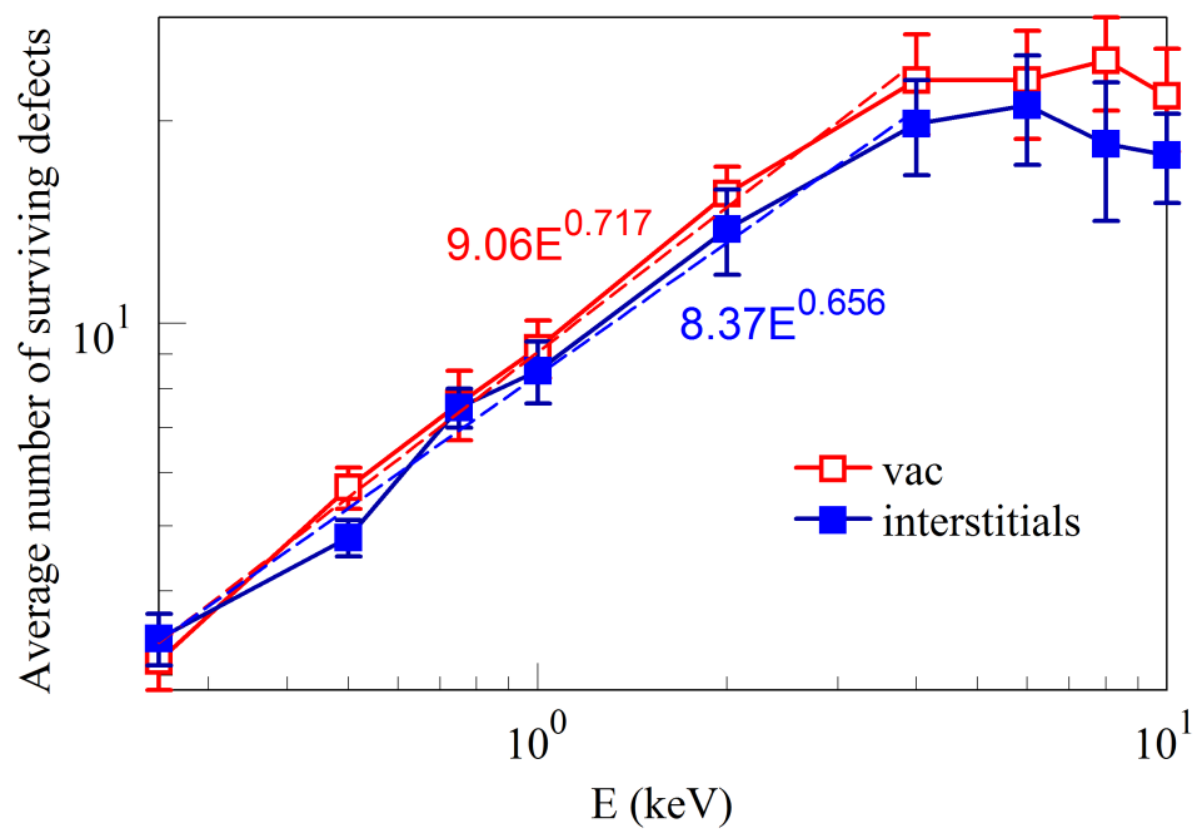

Figure 3. Cascade energy dependence of the surviving defects number for tungsten bombarded by selfatoms of energy E. The average was obtained for the first ten cascades in $300 \mathrm{~K}$ "virgin" tungsten; error bars are the standard errors. While the surviving number follows the NRT power law for energies smaller than $6 \mathrm{keV}$, it saturates at higher energies for the number of cascades considered.

work we extend the study to additional impact energies in the range $250 \mathrm{eV}$ to $10 \mathrm{keV}$, and to room temperature $(300 \mathrm{~K})$ tungsten surfaces. Our goal is to understand the unexpected saturation of the defects obtained previously as well as to check if this saturation persists with change of impact energy. Though hot tungsten is of interest for the next generation machines, we chose here the room temperature for two reasons: 1) ITER will operate at close-to-room temperature coolant [17], and 2) most of the experiments of pre-damaging of tungsten have been done at room temperature $[18,19]$.

The details of our simulation are shown in Section 2, while the obtained results are presented and discussed in Section 3. We give our conclusions in Section 4. 


\section{Details of the simulation}

Single impact MD simulations of displacement damage in bcc and fcc metals has been extensively investigated [11-15]. We extend such studies to simulations of the cumulative effects of displacement damage by following, in addition to the temporal evolution of the damage cascade launched by each self-atom impact, the accumulation of this damage during cumulative impacts of such atoms. We perform our simulations using classical molecular dynamics (MD) simulation code LAMMPS [20] and study the creation and recombination of Frenkel pairs under self-atom impact as well as the evolution of the tungsten surface under cumulative impact of self-atoms. Our bcc tungsten crystal simulation cell is oriented to have a $\langle 100\rangle$ free surface and contains about 26,000 atoms (lateral dimension, $\mathrm{L} \approx 7.6 \mathrm{~nm}$ ) for impact energies $\leq 1 \mathrm{keV}, 128,000$ atoms $(\mathrm{L} \approx 12.7 \mathrm{~nm})$ for 2 and $4 \mathrm{keV}, 351,232$ atoms at $6 \mathrm{keV}(\mathrm{L} \approx 17.7 \mathrm{~nm})$ and 250,000 atoms $(\mathrm{L} \approx 15.8 \mathrm{~nm})$ for $\mathrm{E} \geq 8 \mathrm{keV}$. The size of the numerical cells are chosen so that $99 \%$ of the impact cascade atoms do not reach deeper than half of the cell depth. The two bottom layers of each cell were frozen to avoid slipping of the cell relative to the numerical box due to the momentum it receives by cumulative impacts. The cells are "oversized" to avoid artificial reflection of cascade atoms from the bottom of the cell. The projectile $\mathrm{W}$ atoms were emitted toward the surface from random positions on an emitting surface about $15 \AA$ above the $\mathrm{W}$ surface-vacuum interface, in random directions within $10^{0}$ from normal incidence. The slight variation from perpendicular (z-) impact is to prevent channeling of the impact atoms. 2D periodic boundary conditions were applied (in $\mathrm{x}-\mathrm{y}$ directions).

Each impact at energies $\leq 2 \mathrm{keV}$ was run for $\mathrm{t}=15 \mathrm{ps}$, at larger energies $\mathrm{t}=26 \mathrm{ps}$, with exception of $10 \mathrm{keV}$ which ran for $\mathrm{t}=30 \mathrm{ps}$. These times were found by numerical experimentation, with the goal that the impact cascade ends and any sputtered atom leave the 
numerical box. This part of the evolution performs freely for each impact, without application of any thermostat. The last 6 ps of the impact time was divided between application of a Langevin thermostat ( 5 ps to remove the deposited impact energy, i.e. to reduce temperature to $300 \mathrm{~K}$, followed by 1 ps relaxation time. We used a 0.2 fs time step, which quite well conserved the total energy of the system, in spite of the time discretization.

LAMMPS allows use of the variety of inter-atomic potentials, both many-body and binary interactions. We have selected the Bond Order Potential (BOP), from the well-known family of Tersoff potentials [21] with tungsten parameters developed by Juslin et. al. [22]. The Tersoff potential is the earliest and one of the simplest BOPs (polarization effects are not included, neither is there Coulomb-screening at very short distances). Juslin et al extended this approach in the case of tungsten to include the short-distance universal repulsive potential of Ziegler, Biersack, \& Littmark (ZBL) [23], and to include the effects of second-nearest-neighbors due to the metallic nature of $\mathrm{W}$ atoms bonding. This potential is widely used in MD simulations of tungsten interactions $[5,24]$. It is important to note that this potential permits the defect analysis required for follow-on studies of plasma-surface interactions (possibly including chemistry) with the damaged tungsten. Though there are no chemical reactions in the present system, use of the BOP opens possibilities for future studies of chemically active mixtures of damaged tungsten with carbon, oxygen and hydrogen. We note that our use [25] of the more popular Embedded Atom Method (EAM and its extension MEAM, [26]) resulted in only slightly accelerated calculation time, and produced in the present study virtually identical results to the ones with the BOP potential. 


\section{Simulation Results and Discussion}

The primary damage was produced in displacement cascades by the recoil of primary knock-on atoms and consists of point defects, which cluster. The primary number of vacancies and interstitials in tungsten, as well as their residual number upon recombination, evolve with time, and depend on the impact energy, and temperature of the surface. The number of sequential impacts was about 400 to 500 at all energies.
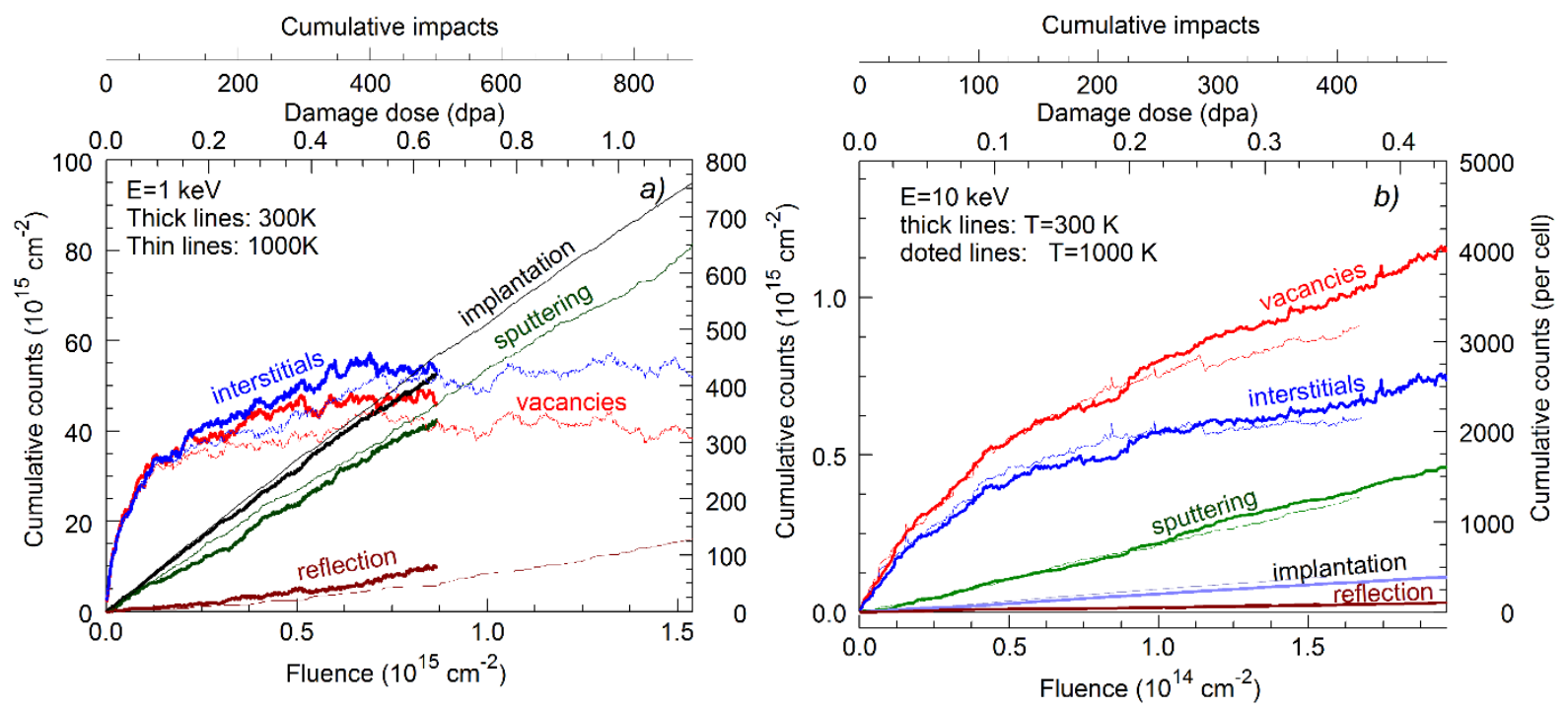

Figure 4. Cumulative counts of the vacancies, interstitials, sputtered, implanted and reflected self-atoms impacting with energy E a mono-crystal of tungsten at $300 \mathrm{~K}$ and $1000 \mathrm{~K}$. Approximate saturations of vacancies and interstitials as function of cumulative number of $\mathrm{W}$ impacts at $\mathrm{E}=1 \mathrm{keV}$ and $10 \mathrm{keV}$ respectively, are visible. Data from Ref. 16 were used for $1 \mathrm{keV}$ at $1000 \mathrm{~K}$.

Figure 4 shows a comparison of defect production, as well as sputtering and implantation, for $300 \mathrm{~K}$ and $1000 \mathrm{~K} \mathrm{~W}$ surfaces at two impact energies. No significant influence of the temperature of the tungsten surface is evident, at least in range $300-1000 \mathrm{~K}$. This may be expected, since the time scale of tens of picoseconds over which the various processes are followed after each impact, is too short for thermal effects to propagate. The trends at the two 
temperatures are very similar, with only minor differences that can be ascribed to random fluctuations. Somewhat smaller sputtering and implantation, and somewhat larger reflection at $300 \mathrm{~K}$ than at $1000 \mathrm{~K}$ for $1 \mathrm{keV}$ can partially explain systematically slightly increased $(<10 \%)$ vacancy and interstitial cumulative counts. At $10 \mathrm{keV}$ the sputtering dominates over the implantation, at all impact fluences, however no temperature effects seems to affect sputtering and implantation. All variations of the cumulative interstitials and vacancies $(<10 \%)$ can be considered random.

We count vacancies by comparison with the unperturbed (undamaged) tungsten cell (the same one which served as a starting target in the cumulative bombardment with self-atoms). Thus, taking the empirical radius of $\mathrm{W}$ atom of $\mathrm{d}=1.36 \mathrm{~A}=\mathrm{a} / 2.33$, where $\mathrm{a}=3.16 \mathrm{~A}$ is the tungsten bcc cell length, we detect a vacancy if there is no atom at a position of a tungsten atom in nondamaged cell and in spherical volume of radius $d$ in an observed instant of time. Similarly, interstitial is detected if an atom in the damaged tungsten is found at a distance larger than $\mathrm{d}$ from any other atom in the non- damaged lattice. Obviously this procedure is not dependent on the level of the system damage.

The dpa values shown in the figures represent the peaks of the vacancy production depth profiles at each W impact energy, obtained using SRIM [23] (Fig. 5) with the Kinchin-Pease As illustrated in our previous publication [16], following each W atom impact an initial "explosion" of a large number of interstitials and vacancies occurs. The collisional cascade cools, and the number of defects stabilizes due to recombination of the vacancies with interstitials and implants, reaching a low, steady-state value within at most a few tens ps, as has been previously observed (e.g., see Fig. 1 in [10]). 


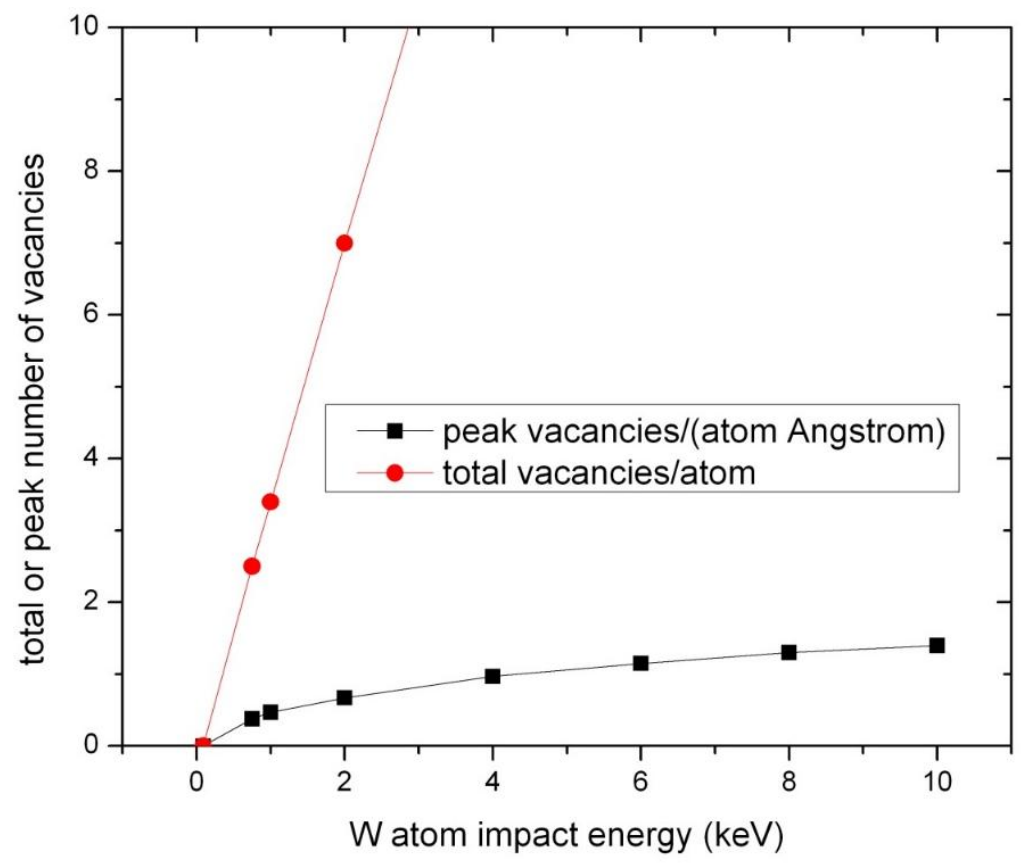

Fig. 5 SRIM results of total and depth resolved vacancy production as function of $\mathrm{W}$ impact energy. formalism [10]. The vacancy production was converted to displacement per atom (dpa) units using the relation: $\mathrm{dpa}=\mathrm{D} \times \Phi / \rho$, where $\mathrm{D}$ is the \# of vacancies/( $\mathrm{\AA} \times$ atom $), \Phi$ is the incident fluence, and $\rho$ is the number density of tungsten $\left(6.33 \times 10^{22} / \mathrm{cm}^{3}\right)$. Savitzky-Golay averaging was applied to the damage profiles to remove near-surface binning irregularities. It was verified that the total vacancy production per ion agreed with the NRT model (eq. 1 in [10]). We used the recommended [27] $90 \mathrm{eV}$ for the threshold displacement damage energy threshold, and SRIM default values [23] of $3 \mathrm{eV}$ for the lattice binding energy, and $8.68 \mathrm{eV}$ for the surface binding energy.

To aid better understanding of the saturation properties of the defect production, and to enable its interpolation/extrapolation to the values of fluences and impact energies not considered here we fit the calculated defect dependences at various impact energies to analytical expressions. Thus, the cumulative sputtering, reflection and implantation numbers $\mathrm{Y}$ are fitted with the straight lines $\mathrm{Y}=\mathrm{DX}$, where $\mathrm{X}$ is the number of cumulative impacts. On the other hand, the saturated dependences, vacancies at impact energies $\leq 1 \mathrm{keV}$ and interstitials at higher energies are fitted to the form $\mathrm{Y}=\mathrm{AX} /(\mathrm{B}+\mathrm{X})$, which saturate to a constant $\mathrm{A}$ at large fluences, $\mathrm{X}>>\mathrm{B}$. Finally, the interstitial dependences at lower energies and vacancy dependences at higher energies are additionally fitted to the form $\mathrm{Y}=\mathrm{AX} /(\mathrm{B}+\mathrm{X})+\mathrm{CX}$, where for $\mathrm{A}$ and $\mathrm{B}$ are used the values obtained for the saturated curves. The conjecture behind this form is that both the number 
of vacancies and interstitials are residuals of the incomplete recombination of the Frenkel pairs created upon the initial impact cascade and that they are therefore approximately equal at

\begin{tabular}{|c|c|c|c|c|c|c|c|c|c|}
\hline \multirow{2}{*}{$\begin{array}{c}\mathrm{E} \\
(\mathrm{keV})\end{array}$} & \multicolumn{3}{|c|}{ Vacancies } & \multicolumn{3}{|c|}{ Interstitials } & \multirow{2}{*}{$\begin{array}{l}\text { Sputt } \\
\text { S*k }\end{array}$} & \multirow{2}{*}{$\begin{array}{l}\text { Impl } \\
I^{*} \mathrm{~K}\end{array}$} & \multirow{2}{*}{$\begin{array}{l}\text { Refl } \\
\mathrm{R} * \mathrm{k}\end{array}$} \\
\hline & A & B & $\mathrm{C}$ & A & $\mathrm{B}$ & $\mathrm{C}$ & & & \\
\hline 0.25 & $\begin{array}{c}181.37 \\
\pm 0.86\end{array}$ & $\begin{array}{l}33.87 \\
\pm 0.98\end{array}$ & $\begin{array}{l}-0.166 \\
\pm 0.001\end{array}$ & $*$ & $*$ & 0.59 & $\sim 0$ & 0.88 & 0.12 \\
\hline 0.50 & $\begin{array}{c}211.46 \\
\pm 0.79\end{array}$ & $\begin{array}{l}21.53 \\
\pm 0.63\end{array}$ & 0 & $*$ & $*$ & 0.51 & 0.25 & 0.85 & 0.14 \\
\hline 0.75 & $\begin{array}{c}361.40 \\
\pm 1.40\end{array}$ & $\begin{array}{l}55.89 \\
\pm 1.00\end{array}$ & 0 & $*$ & $*$ & 0.35 & 0.42 & 0.82 & 0.18 \\
\hline 1 & $\begin{array}{c}410.33 \\
\pm 1.20\end{array}$ & $\begin{array}{l}42.15 \\
\pm 0.67\end{array}$ & 0 & $*$ & $*$ & 0.12 & 0.68 & 0.87 & 0.13 \\
\hline 2 & $*$ & $*$ & 1.06 & $\begin{array}{c}1057.43 \\
\pm 3.05\end{array}$ & $\begin{array}{l}49.09 \\
\pm 0.63\end{array}$ & 0 & $\begin{array}{c}1.68 \\
\pm 0.002\end{array}$ & 0.87 & 0.13 \\
\hline 4 & $*$ & $*$ & 1.84 & $\begin{array}{c}1163.54 \\
\pm 2.67\end{array}$ & $\begin{array}{l}52.68 \\
\pm 0.58\end{array}$ & 0 & $\begin{array}{c}2.30 \\
\pm 0.003\end{array}$ & 0.90 & 0.10 \\
\hline 6 & $*$ & $*$ & 2.06 & $\begin{array}{c}3146.24 \\
\pm 13.29\end{array}$ & $\begin{array}{c}128.91 \\
\pm 1.46\end{array}$ & 0 & $\begin{array}{c}2.38 \\
\pm 0.003\end{array}$ & 0.92 & 0.08 \\
\hline 8 & $*$ & $*$ & 2.65 & $\begin{array}{c}2634.49 \\
\pm 11.52\end{array}$ & $\begin{array}{c}103.72 \\
\pm 1.39\end{array}$ & 0 & $\begin{array}{c}2.79 \\
\pm 0.003\end{array}$ & 0.86 & 0.14 \\
\hline 10 & $*$ & $*$ & 3.05 & $\begin{array}{c}3310.61 \\
\pm 18.26\end{array}$ & $\begin{array}{c}169.45 \\
\pm 2.42\end{array}$ & 0 & $\begin{array}{c}3.22 \\
\pm 0.004\end{array}$ & 0.80 & 0.20 \\
\hline
\end{tabular}

Table I. The fitting coefficients and their standard errors for the defects as function of the number of cumulative impacts, $\mathrm{k}$, of self-atoms of kinetic energy $\mathrm{E}$ on a $<100>$ tungsten mono crystal of temperature $300 \mathrm{~K}$. The cumulative vacancies and interstitials are fitted to the form $\mathrm{A} k /(\mathrm{B}+k)+\mathrm{C} k$, while cumulative sputtering, implantation and reflection are fitted to the straight lines Sk, Ik, and Rk, respectively. Conversion factor of the impact number to the fluence depends on the interface surface area, and is $1.73 \times 10^{12} \mathrm{~cm}^{-2}$ for $\mathrm{E} \leq 1 \mathrm{keV}, 6.23 \times 10^{11}$ for 2 and $4 \mathrm{keV}, 3.23 \times 10^{11}$ for $6 \mathrm{keV}$, and $3.99 \times 10^{11}$ for 8 and $10 \mathrm{keV}$.

fluences low enough to neglect effects of sputtering and interstitials. At higher fluences, as discussed below, the increase of interstitials (at low energies) and vacancies (at higher energies) 
is governed by the linear increases of cumulative implantation and sputtering numbers, respectively. We list all obtained coefficients in Table I.

Both implantation and sputtering play important roles in the final balance of the defects, however their mutual influence is not the same at all impact energies. Typical cases for $\mathrm{E} \leq 1$ $\mathrm{keV}$ are shown in Figure 7. At $\mathrm{E}=250 \mathrm{eV}$ sputtering is negligible, and the large number of interstitials is a reflection of the dominance of implantation $\left(\mathrm{P}_{\mathrm{impl}}=0.88\right.$ implants $/ \mathrm{W}$, practically all that are not reflected, $\left.\mathrm{P}_{\text {ref }}=0.12 / \mathrm{W}\right)$. The interstitial curve approximately follows the slope of the implantation line at large cumulative impact number. As the number of implants increase, the number of vacancies decreases due to the increased recombination with implanted W's. At 500 eV impact energy, all impact (except reflected) atoms are implanted, however the sputtering probability of $\mathrm{P}_{\text {sput }}=0.25 / \mathrm{W}$ leads to creation of additional vacancies which recombine with implants, keeping the number of vacancies almost constant. This trend is seen also at $\mathrm{E}=0.75$
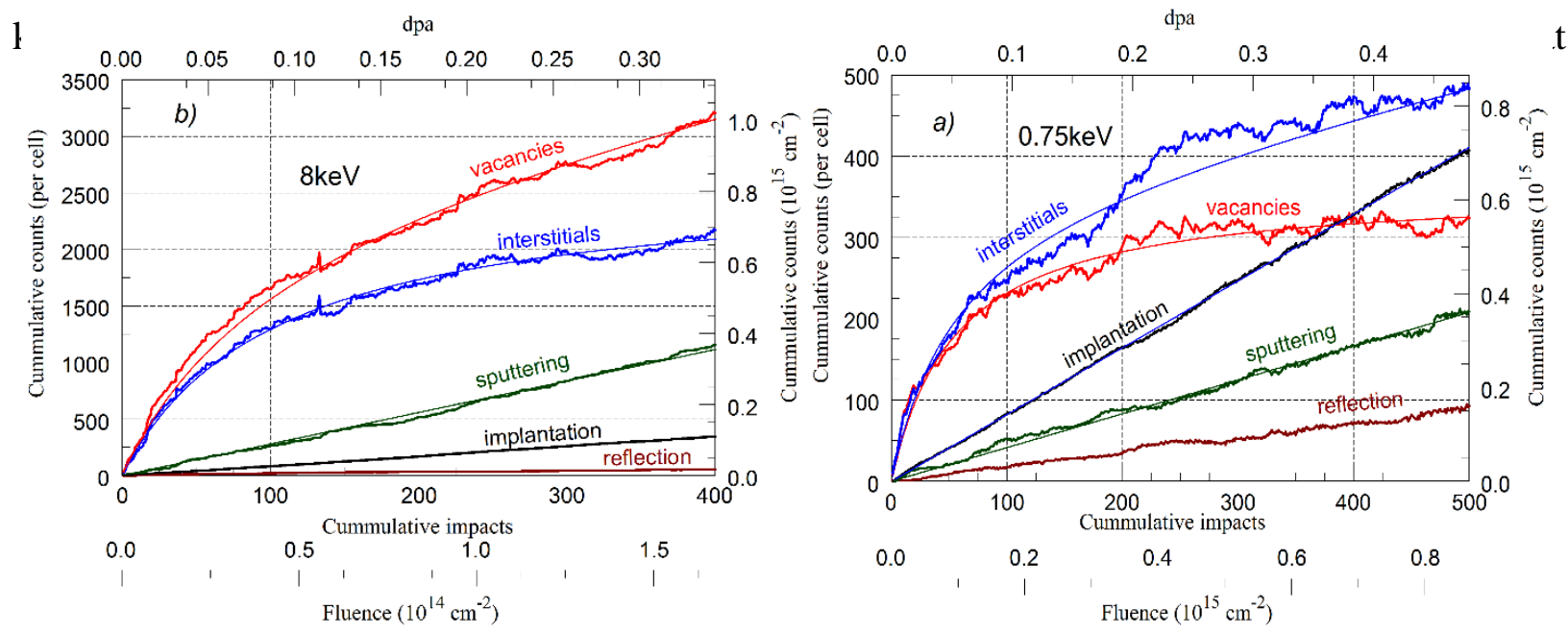

Figure 6. Typical fitting curves (thin lines), defined in Table I for two impact energies, $0.75 \mathrm{keV}$ and $8 \mathrm{keV}$. 
these energies is close to the difference of implanted and sputtered atoms. Thus, the dashed line in Fig. $7(b)$ is obtained by adding $\triangle(i, v)$ to the cumulative vacancy curve.

At all studied impact energies, many of the vacancies and interstitials from the initial damage recombine, resulting in a relatively small number of final defects, whose number saturates after a few hundreds of impacts at $\mathrm{E} \leq 1 \mathrm{keV}$ and after a few thousands at $\mathrm{E} \geq 2 \mathrm{keV}$. A significant number of the primary vacancies are created by sputtering which subsequently recombine with implanted self-atoms as long as the implantation production is larger than the vacancy one. This changes at higher energies, as shown in Fig. 8. The dominance of interstitials over vacancies is due to the dominance of sputtering vs. implantation. The interstitial number flattens at larger number of cumulative impacts, since most of the implants recombine with a part of vacancy count created by sputtering. As a consequence, the difference between the dominant number of defects (here vacancies) and minority defects (here interstitials) is close to
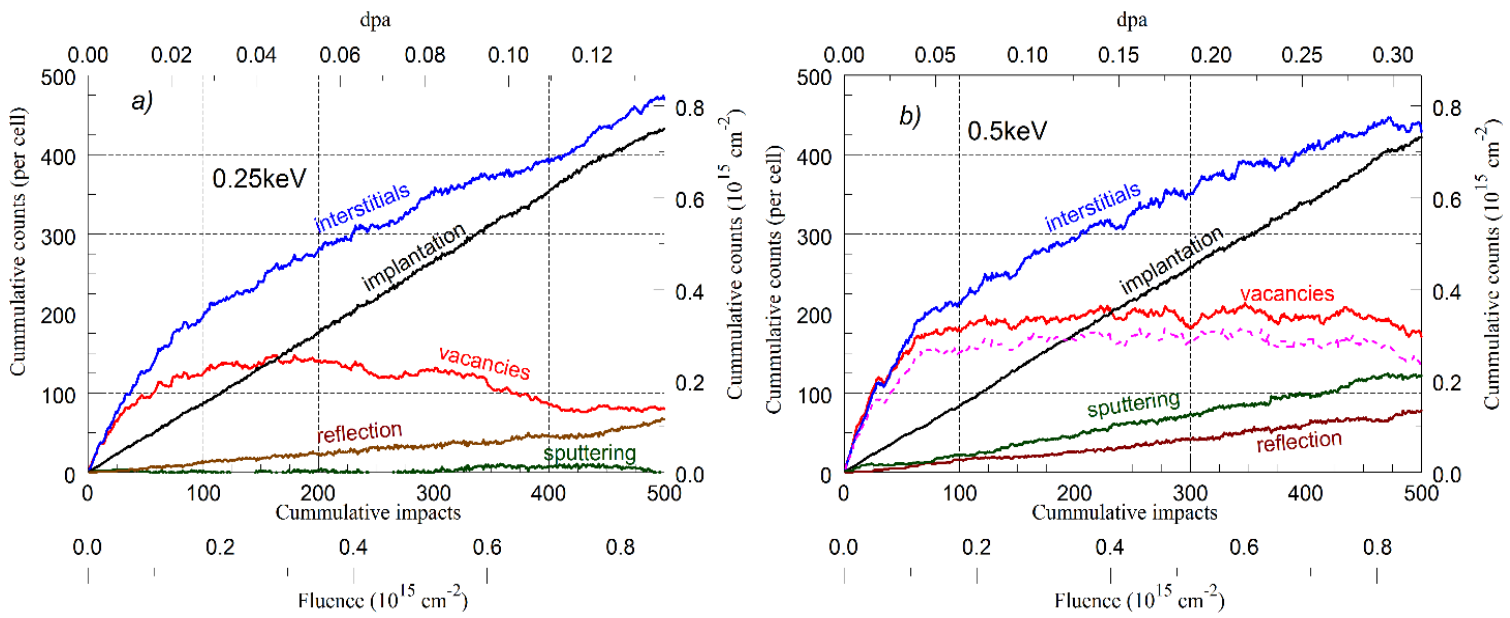

Figure 7. Cumulative defects at impacts energies $\mathrm{E} \leq 1 \mathrm{keV}, \mathrm{T}=300 \mathrm{~K}$. a) $\mathrm{E}=250 \mathrm{eV}$; b) $\mathrm{E}=500$ $\mathrm{eV}$.

the difference between cumulative number of sputtered and implanted W. The dashed curves in 8b) and 8c) as well as in Fig. 7b) are obtained by adding the difference of cumulative number of 
sputtered and implanted $\mathrm{W}$ to the number of interstitials. Obviously, $\triangle(\mathrm{i}, \mathrm{v})$ is a negative number at all considered fluences for all cases in Fig. 7.
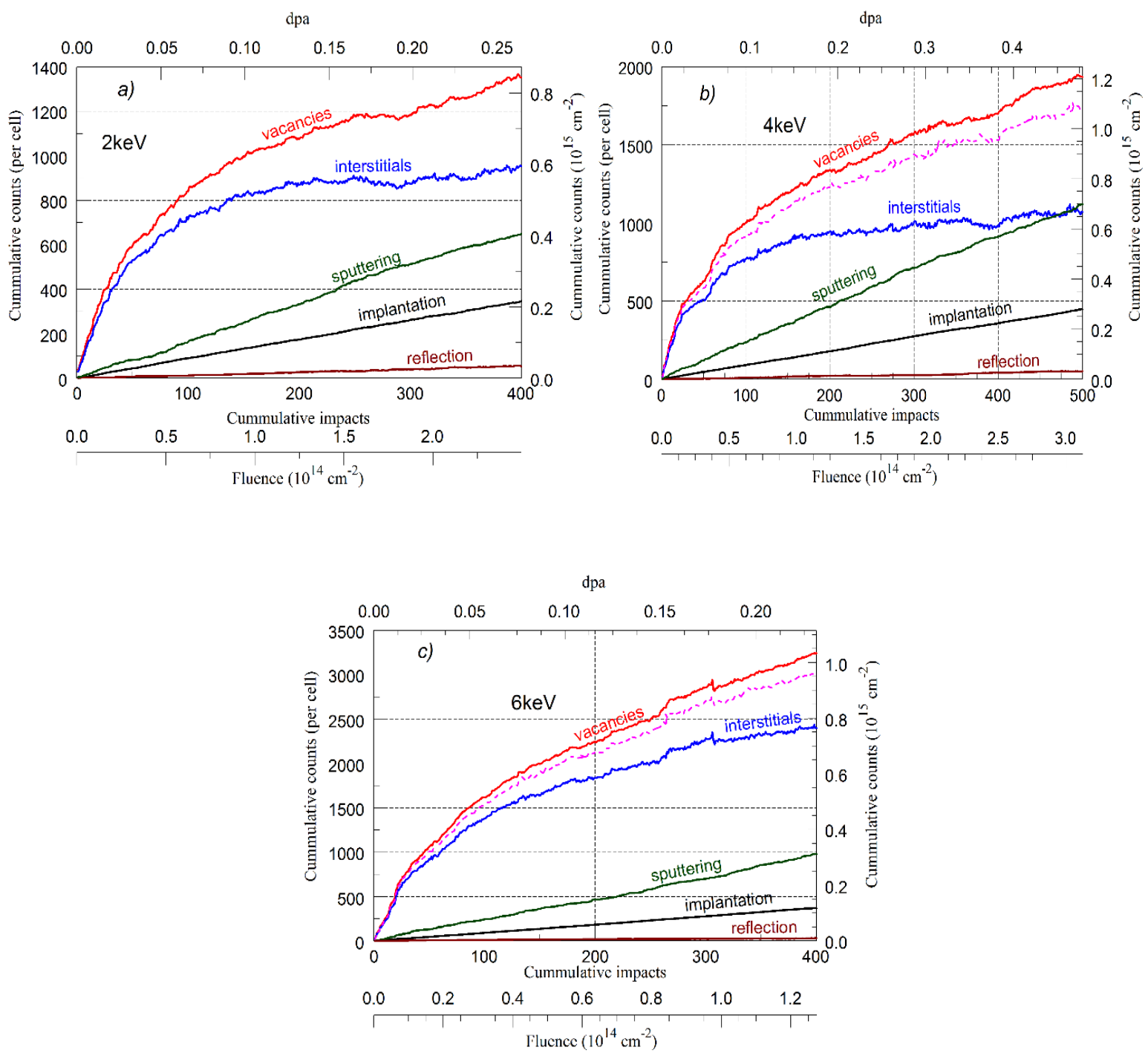

Figure 8. Cumulative defects at impacts energies $\mathrm{E} \geq 1 \mathrm{keV}, \mathrm{T}=300 \mathrm{~K}$.

As seen in table I, and shown in Figure 9 the implantation fraction is close to 1 and almost independent of energy, while the sputtering yield strongly increases with energy. Although smaller than the implantation count at $\mathrm{E} \leq 1 \mathrm{keV}$, the sputtering count significantly exceeds it at higher energies. The obtained sputtering yields are in excellent agreement with published values [28]. Crossing of the curves in Fig. 9 for sputtering and implantation is seen 
between 1 and $2 \mathrm{keV}$ impact energy. The implantation $(>0.8)$ and reflectance $(<0.2)$ probabilities/W vary little in the whole range of energies. The sputtering yield/W increases from 0.25 at $250 \mathrm{eV}$, to larger than $1(\sim 1.7)$ at $2 \mathrm{keV}$ impact energy, and then changes slowly with energy (reaching $\sim 3.2$ at $10 \mathrm{keV}$ ), staying larger than 1 .

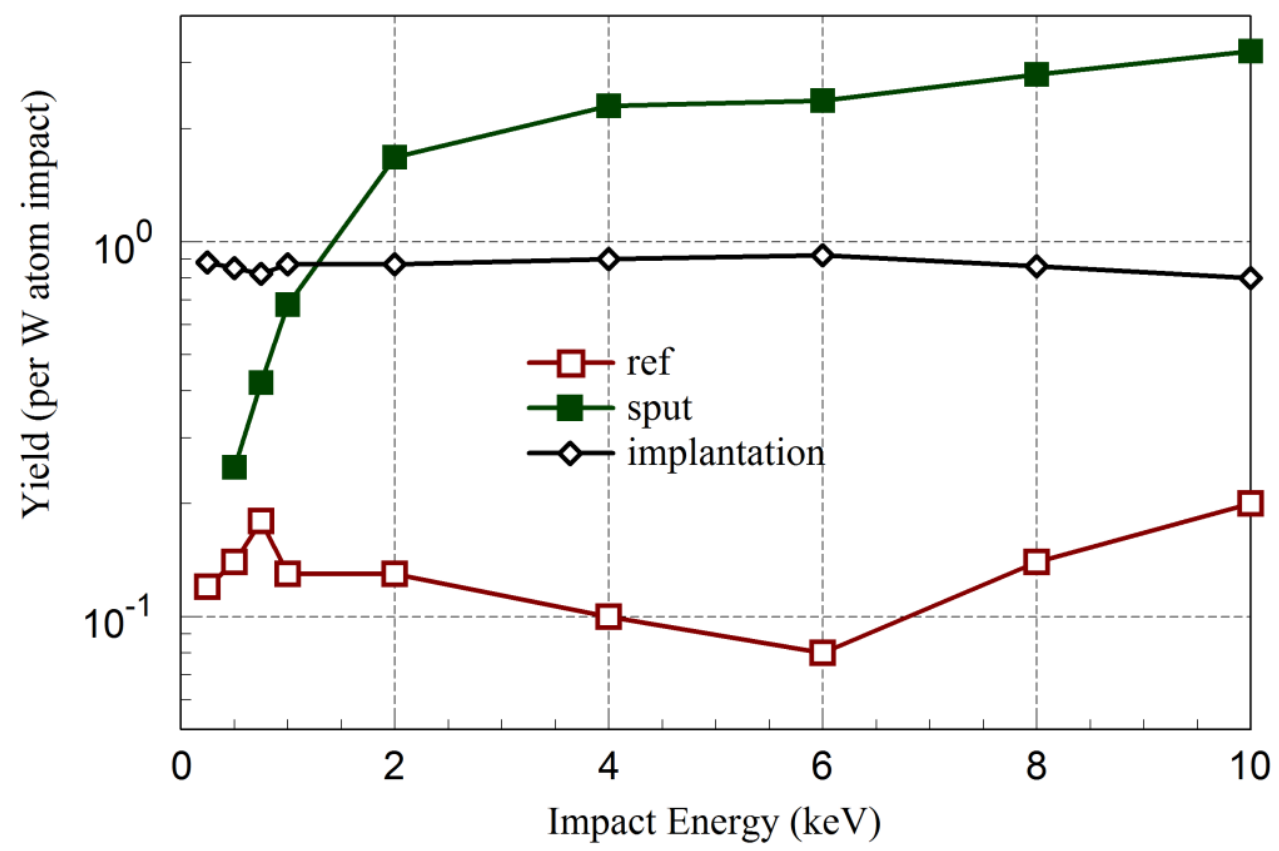

Figure 9. Dependence of sputtering, implantation and reflection per impacting $\mathrm{W}$ atom, obtained from the slopes of the relevant straight line fits in Table I.

For lower energies the peak steady-state defect level of vacancies is reached between 0.02 and $0.1 \mathrm{dpa}$. At higher energies, the number of vacancies never fully saturates but slowly linearly increases with the sputtering count (minus implantation). This regime is reached at about $0.1 \mathrm{dpa}$ for impact energies larger than $1 \mathrm{keV}$. The increase is still much slower than the increase of the vacancies from the virgin surface, for the lowest fluences. The simulation results thus confirm our earlier conjecture that the final material damage level is significantly lower than the external self-ion damage dose. The much slower increase of the damage level at the higher 
energies could be partly responsible for the recently reported [18,19] saturation of H-retention in room temperature $\mathrm{W}$ at self-ion damage doses above $1 \mathrm{dpa}$, obtained by MeV's impacts of selfions.

For completeness, in Figure 10 we present the dependences of the cumulative vacancies and interstitials as function of impact energy, for three distinct values of the impact fluence. The values for higher impact energies at fluences of $7 \times 10^{14} \mathrm{~cm}^{-2}$ and $2 \times 10^{15} \mathrm{~cm}^{-2}$ are obtained by extrapolation of the fitting formulas in Table I. The transition from the dominant number of interstitials to the dominant number of vacancies is visible at impact energies slightly larger than $1 \mathrm{keV}$, for all three cases of impact fluences. It is interesting that the cumulative interstitial counts tend to constant values close to $10^{15} \mathrm{~cm}^{-2}$ with increasing energy and impact fluence. On the other hand, the cumulative vacancy count reaches a power law dependence on energy, i.e. it behaves approximately as $\mathrm{E}^{0.6}$. This is similar to the fit of the dependence of the $\mathrm{C}$ parameters for vacancies in Table I, which gives $\mathrm{C} \sim \mathrm{E}^{0.6}$. This indicates that for large fluences $\left(\mathrm{F}>5 \times 10^{14} \mathrm{~cm}^{-2}\right)$ and higher energies the cumulative vacancy count created by self-atom impact increases as $\mathrm{FE}^{0.6}$.

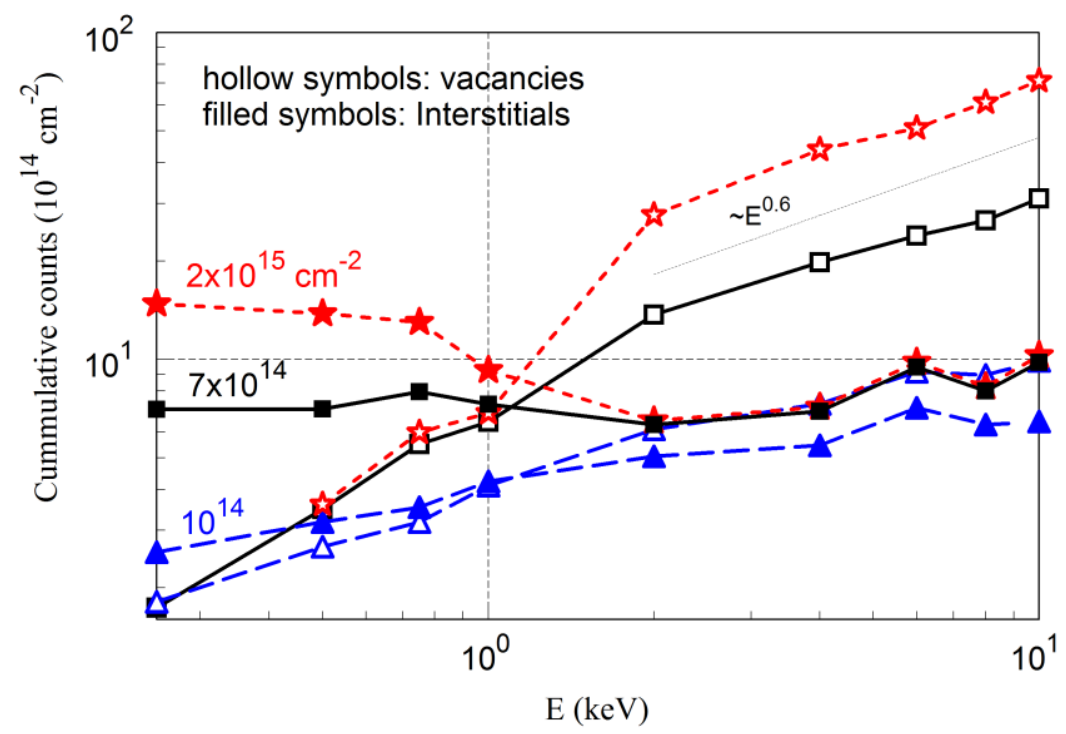

Figure 10. The impact energy dependence of the cumulative number of vacancies and interstitials at three fixed fluences of $\mathrm{W}$. 
For all considered energies most of the produced defects tend to accumulate close to the surfacevacuum interface, reflecting damage of the first few layers of the surface. As illustration, we show in Figure 11 the defect distributions at impact energies of $1 \mathrm{keV}$ and $6 \mathrm{keV}$ for a damage dose of about $0.6 \mathrm{dpa}$ and $0.25 \mathrm{dpa}$, respectively. We record the defects in $1.58 \AA$ bins, above and below the interface. For $6 \mathrm{keV}$ the vacancy distribution extends to about $20 \AA \sim 13$ unperturbed atomic layers or lattice planes) in depth and is strongly peaked at the interface. Significant numbers of interstitials accumulate as adatoms up to approximately $5 \AA$ above the original

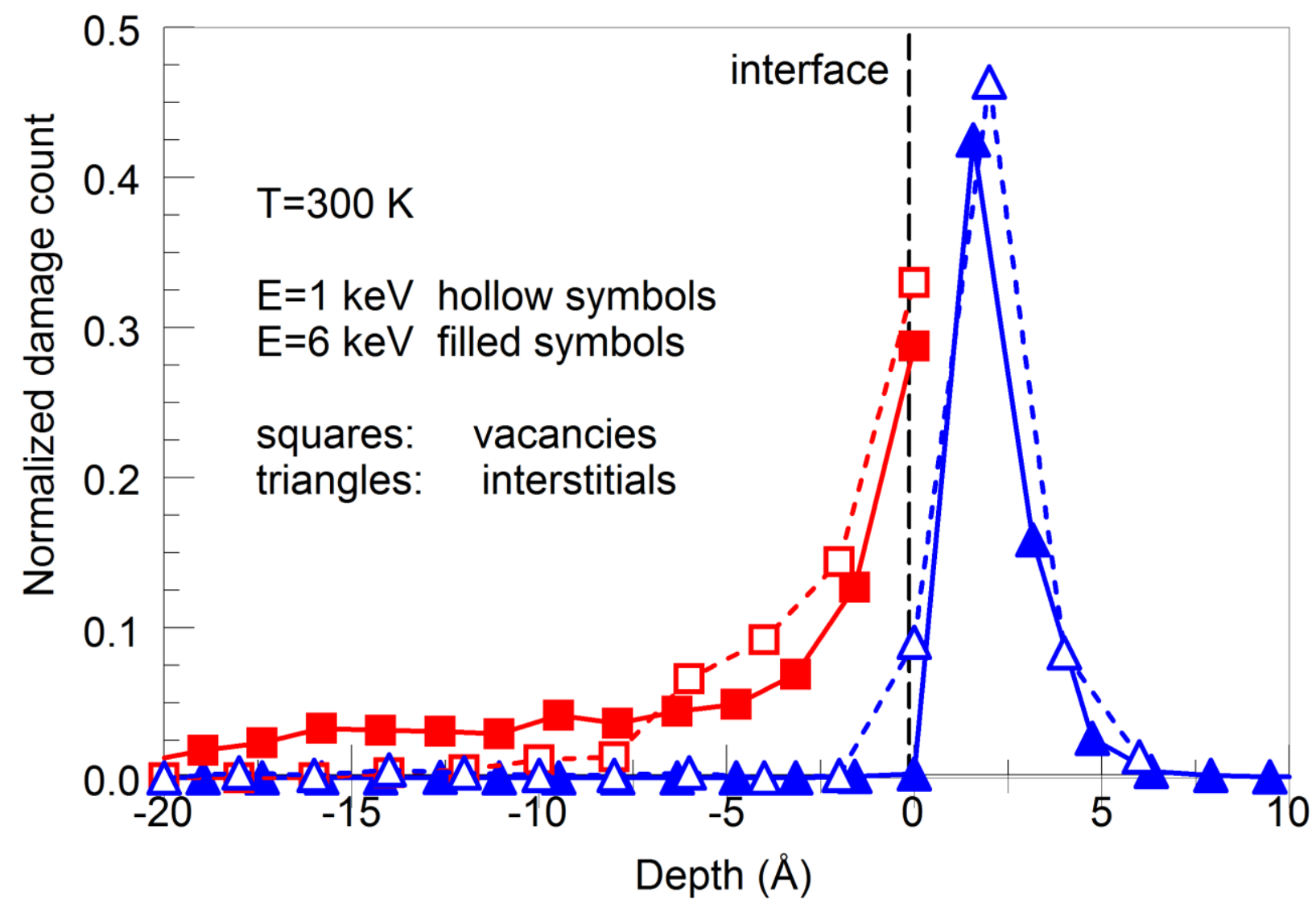

Figure 11. Depth distribution of normalized cumulative impact count, after 500 impacts, induced by W self-atom impact at the indicated energies and temperature. Note the unperturbed surface is at 0 angstrom. Positive depths are above the initial, unperturbed vacuum-surface interface. The above-surface interstitials, shown at positive depths, correspond to what are conventionally referred to as adatoms. The normalization of damage counts is to the total initial unperturbed $\mathrm{W}$ atom number per layer. The symbols are at the centers of the bins of $1.58 \AA$ width in z-direction, positioned at the unperturbed layers of W. 
interface, contributing together with the first-layer vacancies to surface roughening rather than being traps for $\mathrm{H}$ and $\mathrm{He}$ atoms.) The percentages of adatoms in interstitials as function of impact energy E are presented in Figure 12.

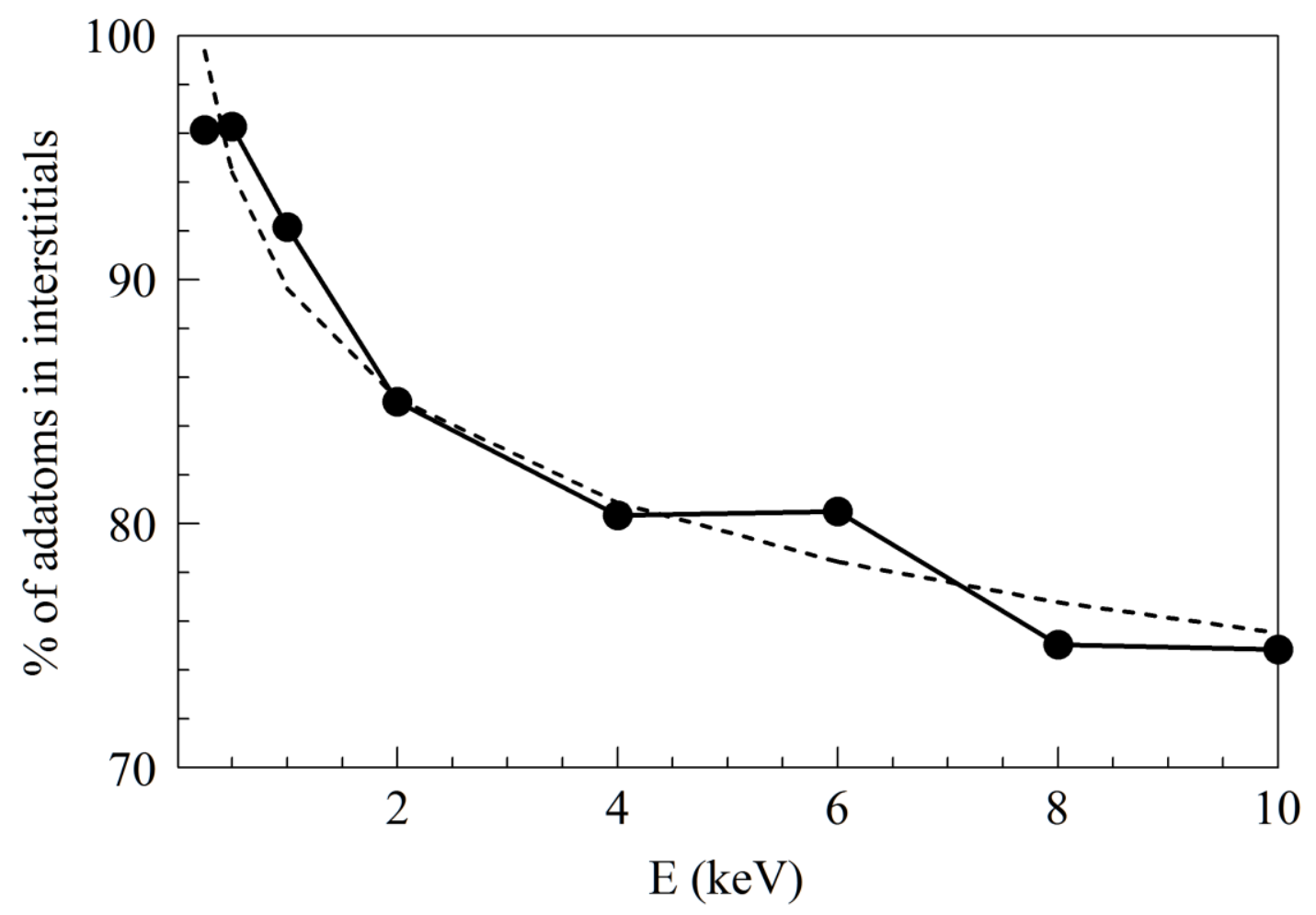

Figure 12. Percentage of adatoms in interstitials, $I_{A}$, at various impact energies $\mathrm{E}$. For $\mathrm{E}>0.5 \mathrm{keV}$, this dependence is well fitted by a power function $I_{A}=a E^{-b}$, where $a=89.64 \%$ and $b=7.44 \times 10^{-2}$. As expected, at low impact energies almost all interstitials are adatoms, while their percentage decreases at higher energies, reaching about $75 \%$ at the $10 \mathrm{keV}$.

Other effects of nearby surfaces on the evolution of displacement damage cascades has been have been reported in earlier MD simulation studies [11-15]. Interestingly, vacancies initially created deeper in the bulk as steady-state Frenkel pairs are mainly filled by implants. Thus, implantation has a role in narrowing of the vacancy distribution toward the surface. 


\section{Conclusions}

We carried out damage simulations that account for the material response to the damage dose in detail, for a range of energies and for room temperatures, which are mainly used in the experiments. From the MD simulation results in Section 3, it is evident that the self-atom damage quickly saturates at lower energies with increase of fluence. This reduces the defect density in the near-surface region (where He can be trapped), opposite to linear increase with fluence of the damage dose obtained from SRIM. The saturation is a result of the recombination of vacancies and interstitials that lie within a critical distance of each other. This saturation behavior is evident for vacancies in MD simulations carried out in impact energy range $0.25-1$ $\mathrm{keV}$ and for interstitials in range $2-10 \mathrm{keV}$, but is expected to be relevant for interstitials at energies higher than those calculated here as well. As was discussed in [10] in connection with $\mathrm{H}$ retention in $\mathrm{W}$ pre-damaged by MeV-energy $\mathrm{W}$ self-ions [10], when the self-atom impact damage accumulation data are used to estimate neutron damage at the relevant energies, the implantation yield has to be added to the vacancy cumulative count numbers, since neutrons cannot be used to recombine the vacancies created by sputtering. Our counting finds that the implanted atoms contribute only a small fraction of the interstitial defects (too small to be shown in the figures).

Sputtering, implantation and reflection are linear functions of the impact number in the considered range of fluences and energies. The efficiency of implantation is more than $80 \%$ per impact atom at all energies, and most of the implanted atoms ( 95\%) end up at vacancy sites, thus reducing the total number of vacancies remaining after the "pacification" of each collision cascade in comparison to that from the steady-state Frenkel pair count. The competing process, i.e. sputtering, is less efficient than implantation at impact energies below $1 \mathrm{keV}$, but it becomes 
dominant over implantation at higher energies, becoming a significant contributor to the vacancy count. Sputtering produces vacancies, but in fewer numbers than their reduction due to implants that end up at vacancy sites. The difference of the number of interstitials and vacancies almost exactly reproduces the difference of implantation and sputtering counts.

\section{Acknowledgements}

Y Wu acknowledges the supports of the National Basic Research Program of China under Grant No. 2013CB922200 and of National Natural Science Foundation of China under Grant No. 11474032. PSK acknowledges support of the LDRD program of Princeton Plasma Physics Laboratory, managed by Princeton University, for the U.S. Department of Energy. FWM and PSK acknowledge support of the LDRD Program of Oak Ridge National Laboratory, managed by UT-Battelle, LLC, for the U.S. Department of Energy. PSK acknowledges allocation of advanced computing resources provided by the National Science Foundation and U.S. Department of Energy, as well as of Handy computing cluster of Institute of Advanced Computational Science of the Stony Brook University. YW and FYZ acknowledge use of the "Dawning" supercomputer of the Institute of Applied Physics and Computational Mathematics, Beijing, China.

\section{References}

[1] S. Takamura, N. Ohno, D. Nishijima and S. Kajita, Plasma Fusion. Res. 1 (2006) 051.

[2] M. J. Baldwin and R. P. Doerner, Nucl. Fusion 48 (2008) 035001.

[3]T. Tanabe, Phys. Scr. T159 (2014) 014044.

[4] K. O. E. Henriksson, K. Nordlund, A. Krasheninnikov and J. Keinonen, Fusion Science and Technology 50 (2006) 43.

[5] X. Yang, A. Hassananein, J. Nucl. Mat. 434 (2013) 1. 
[6] X. Yang, A. Hassananein, Nucl. Instr. Meth. Phys. Res. B 308 (2013) 80.

[7] A. Ito, Phys. Scr. T159 (2014) 014062.

[8] M. J. Baldwin, R. P. Doerner, J Nucl Mater 404 (2010) 165-173.

[9] F. W. Meyer, H. Hijazi, P. S. Krstic, S. Dadras, H. M. Meyer III, C. M. Parish, M. E. Bannister, Phys. Scr. T159 (2014) 014029.

[10] R. E. Stoller, M. B. Toloczko, G. S. Was, A. G. Certain, S. Dawarasknath, and F. A. Garner, Nucl. Instrum. Meth. B 310 (2013) 75.

[11] Mai Ghaly and R.S. Averback, Physical Review Letters 72, 364 (1994).

[12] D. J. Bacon, F. Gao, and Yu. N. Osetsky, J. Nuclear Materials 276, 1 (2000).

[13] K. Nordlund, J. Keinonen, M. Ghaly and R. S. Averback, Nature 398, 49 (1999).

[14] R. E. Stoller, J. Nuclear Materials 276, 22 (2000).

[15] A. F. Calder, D. J. Bacon, A. V. Barashev, and Yu. N. Osetsky, Philosophical Magazine 90, 863 (2010).

[16] F. W. Meyer, P. S. Krstic, H. Hijazi, M. E. Bannister, J. Dadras, C. M. Parish and H. M. Meyer III, IOP Conf. Ser. 488 (2014) 012036.

[17] S. J. Ployhar, B. Gopalapillai, L. C. Teodoros et al, Fusion Eng. Des 89 (2014) 1975.

[18] M. H. J 't Hoen, B. Tyburska-Püschel, K. Ertl, M. Mayer, J. Rapp, A. W. Kleyn and P. A. Zeijlmans van Emmichoven Nucl.Fusion 52 (2012) 023008.

[19] G. M. Wright, M. Mayer, K. Ertl, G. de Saint-Aubin and J. Rapp Nucl. Fusion 50 (2010) 075006.

[20] S. Plimpton, Fast Parallel Algorithms for Short-Range Molecular Dynamics, J Comp Phys, 117 (1995) 1-19.

[21] J. Tersoff, Phys. Rev. B 37 (1988) 6991.

[22] N. Juslin, P. Erhart, P. Traskelin et al, J. Appl. Phys. 98 (2005) 123520.

[23] J. F. Ziegler, J. P. Biersack and U. Littmark TRIM (transport and range of ions in matter) The Stopping and Range of Ionsin Solids ed J. F. Ziegler, J. P. Biersack and U. Littmark (New York: Pergamon Press 1985).

[24] X. Yu, F. Gou, and X. Tian, J Nucl Mater 441 (2013) 324.

[25] P.S. Krstic and J. Dadras, Unpublished results \{2014): The comparison was done with 1 $\mathrm{keV}$ and $10 \mathrm{keV} \mathrm{W}$ cumulative bombardment of the tungsten surface.

[26] A. Moitra, S. Kim, J. Houze et al, J. Phys. D: Appl. Phys. 41(2008) 185406. 
[27] ASTM E521, Standard Practice for Neutron Radiation Damage Simulation by Charged

Particle Irradiation, Annual Book of ASTM Standards, Vol 12.02, (ASTM International, West Conshohocken, PA, 2009).

[28] W. Eckstein, Top. Appl. Phys. 110: Sputtering by Particle Bombardment (Berlin: Springer, 2007). 
Cumulative impacts

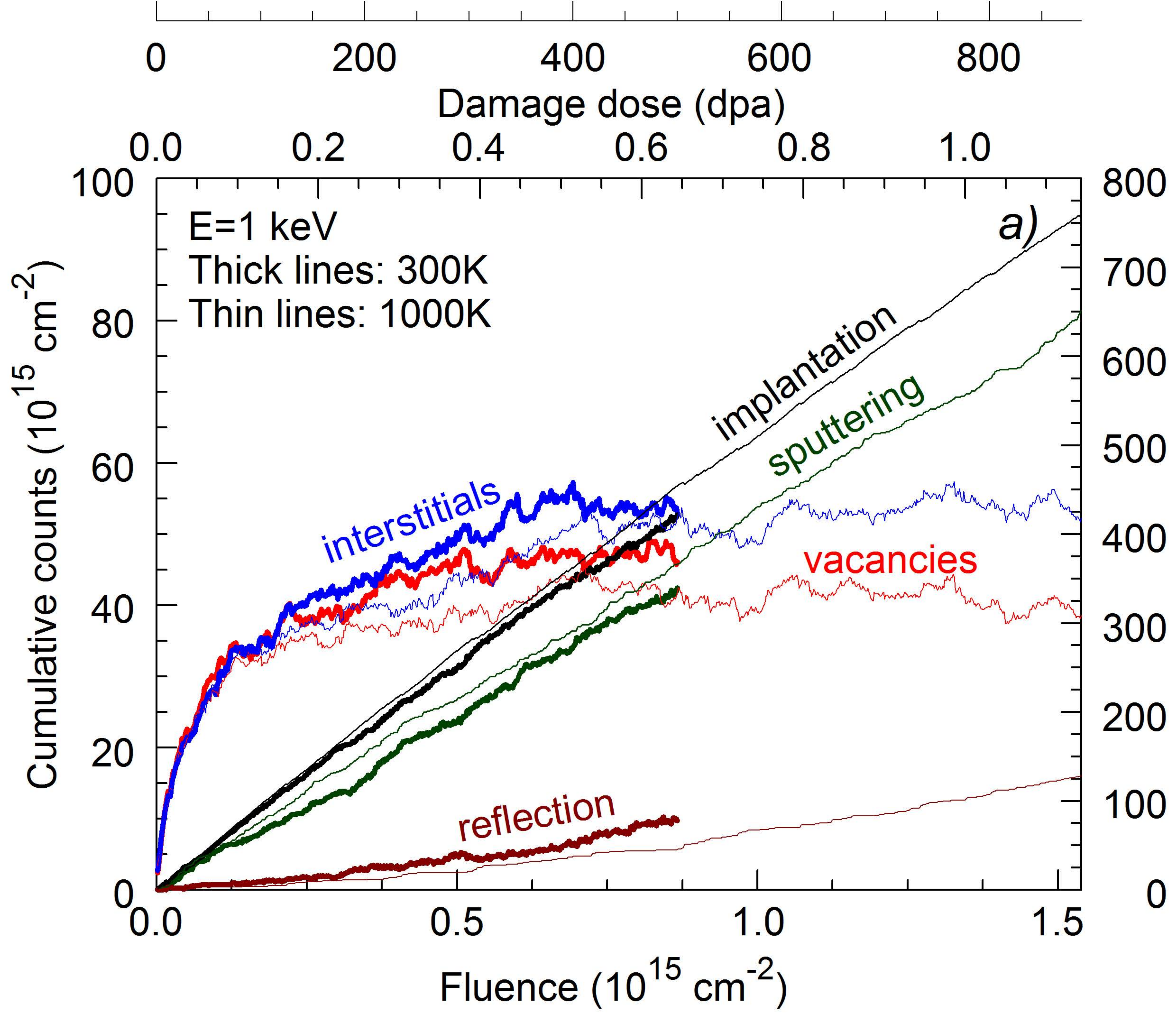

